\title{
Comparison of Prothrombin Time and Aspartate Aminotransferase in Predicting Hepatotoxicity After Acetaminophen Overdose: a Response
}

\author{
Michael Levine ${ }^{1,2} \cdot$ Ayrn D. O'Connor ${ }^{2} \cdot$ Angela Padilla-Jones $^{2} \cdot$ Richard Gerkin $^{2}$
}

Published online: 13 November 2015

(C) American College of Medical Toxicology 2015

To the Editor,

We would like to thank Drs. Hernandez and Nelson for their comments on our manuscript and are thankful for the opportunity to address each of their concerns. Regarding the difficulty in discerning the research question, as was stated in the Abstract and Background sections of the manuscript, the primary objective was to compare the test characteristics of the initial aspartate aminotransferase (AST) and prothrombin time (PT) in patients treated for acetaminophen overdose [1]. A secondary objective was to better characterize coagulation abnormalities in acetaminophen toxicity, as there is little in the medical literature that describes changes in PT early in the course of acetaminophen overdose. And although Drs. Hernandez and Nelson are right to point out that the timing of exposure is important, we felt that reliability of the history provided regarding this timing is highly variable and therefore we maintained focus on comparing initial AST and PT relative to each other.

We agree with Drs. Hernandez and Nelson that including patients with initial AST $>1000 \mathrm{IU} / \mathrm{L}$ would be a significant confounder in evaluating the prognostic utility of initial AST. This was the very reason that the test characteristics for initial AST, initial PT, and maximal PT were

Michael Levine

Michael.Levine@bannerhealth.com

1 Section of Medical Toxicology, Department of Emergency Medicine, University of Southern California, Los Angeles, CA, USA

2 Department of Medical Toxicology, Banner Good Samaritan Medical Center, Phoenix, AZ, USA calculated for the 247 patients who did not have hepatotoxicity on presentation. The text in the Results section and Table 2 highlight that the 57 patients who presented with AST $>1000 \mathrm{IU} / \mathrm{L}$ were removed from the calculations in determining the test characteristics and only patients with AST $<1000$ IU/L were included.

Drs. Hernandez and Lewis raise further concerns regarding Table 1 and the difference between the mean and median initial AST. Both the mean with standard deviation and median with interquartile range were provided to highlight for the reader that indeed the data are non-normalized. As one would expect, those patients with fulminant hepatic failure who are the sickest would skew the data.

Our overall hope for this study and manuscript was to provide data regarding PT, specifically quantitating its test characteristics which are scarcely available in the literature that characterizes acetaminophen toxicity. We also wanted to take the opportunity to discuss factors that affect the PT, its interpretation, and its potential use as a prognostic indicator. Specifically, acetaminophen's ability to inhibit gamma-carboxylation of vitamin K-dependent clotting factors and IV NAC's ability to cause mild elevations in PT were highlighted. Thank you for your interest in our study and we hope this has helped to clarify any confusion.

\section{Reference}

1. Levine M, O'Connor AD, Padilla-Jones A, Gerkin RD. Comparison of prothrombin time and aspartate aminotransferase in predicting hepatotoxicity after acetaminophen overdose. J Med Toxicol. 2015; published online 04 September:1-7. 